\title{
ANÁLISE DOS FATORES IMPLICADOS NA CONVERSÃO DA COLECISTECTOMIA LAPAROSCÓPICA
}

\section{FACTORS IMPLIED WITH CONVERSION TO OPEN CHOLECISTECTOMY}

\author{
Enio Corrêa Lima ${ }^{1}$; Fábio Lopes Queiroz ${ }^{2}$; Felipe Neto Ladeira ${ }^{3}$; \\ Bruno Meilman Ferreira³; José Guilherme Penido Bueno ${ }^{3}$; Eudes Arantes Magalhães, ACBC-MG ${ }^{4}$
}

\begin{abstract}
RESUMO: Objetivo: Analisar os fatores implicados na conversão da colecistectomia laparoscópica. Método: Análise retrospectiva dos protocolos dos pacientes submetidos a colecistectomia laparoscópica no período de janeiro de 2000 a dezembro de 2004. Resultados: Foram realizadas 1734 colecistectomias laparoscópicas neste período. A taxa de conversão foi de 5,7\%, sendo de $5,44 \%$ nas cirurgias eletivas e de $14 \%$ nas cirurgias de urgência. $(\mathrm{p}=0,019)$. Não houve diferença na taxa de conversão em relação ao sexo, 7,46\% nos pacientes do sexo masculino e 5,39\% do sexo feminino. ( $\mathrm{p}=0,178$ ). A conversão foi necessária em $4,41 \%$ dos pacientes com menos de 60 anos e em $11,11 \%$ dos pacientes maiores de 60 anos $(\mathrm{p}=0,01)$. O principal motivo responsável pela conversão foi inflamação e aderência entre as estruturas do pedículo biliar $(42,53 \%)$, outras causas foram coledocolitíase $(17,24 \%)$ e suspeita ou lesão da via biliar $(9,19 \%)$. Um tempo operatório maior que 120 minutos foi observado em $71,43 \%$ dos pacientes convertidos, e em apenas $8,2 \%$ dos pacientes não-convertidos. Dos pacientes convertidos $79,31 \%$ tiveram alta após 48 horas, e entre os pacientes não-convertidos apenas $10,8 \%$ tiveram alta após este período.( $\mathrm{p}=0,001)$. Conclusão: Os fatores de risco para conversão observados neste estudo foram idade> 60 anos, cirurgia em caráter de urgência e pacientes com colecistite aguda (Rev. Col. Bras. Cir. 2007; 34(5): 321-325).
\end{abstract}

Descritores: Colecistectomia; Laparoscopia; Fatores de risco.

\section{INTRODUÇÃO}

Estima-se que atualmente $90 \%$ das colecistectomias sejam realizadas pela técnica laparoscópica, percentual este atingido nos Estados Unidos da América no ano de 1992.

Os motivos para tal preferência na escolha da técnica cirúrgica aplicada são claros: menor dor no pós-operatório, recuperação pós-cirúrgica mais rápida, menor número de dias de trabalho perdidos e menor tempo de permanência hospitalar ${ }^{1}$.

A colecistectomia laparoscópica foi claramente estabelecida como padrão-ouro para o tratamento cirúrgico da litíase biliar, no entanto 2 a $15 \%$ das colecistectomias vídeolaparoscópicas necessitam de conversão para cirurgia convencional, sendo as razões mais comuns a inabilidade para se identificar corretamente a anatomia, suspeita de lesão da árvore biliar e sangramento ${ }^{2}$.

O objetivo deste trabalho é analisar a taxa de conversão das colecistectomias vídeo-laparoscópicas e os fatores implicados na mesma.

\section{MÉTODO}

Foi realizado estudo retrospectivo, analisando-se 1922 protocolos dos pacientes submetidos a colecistectomia no Hospital Governador Israel Pinheiro, entre janeiro de 2000 a novembro de 2004. Destas 1922 colecistectomias realizadas, 1734 foram realizadas pela via laparoscópica, 62 por laparotomias e oito por minilaparotomia. Foram excluídos 118 protocolos por apresentarem dados inconsistentes. A escolha da técnica aberta por laparotomia ou mini-laparotomia ocorreu de acordo com a preferência do cirurgião.

Em 99 pacientes foi necessária a conversão para cirurgia aberta.

As variáveis analisadas foram: taxa de conversão, tempo cirúrgico, motivo da conversão, cirurgia em caráter eletivo ou não, idade do paciente, sexo, ASA (risco cirúrgico segundo a American Society of Anesthesiology), complicações pós-operatórias e a duração da internação.

$\mathrm{Na}$ análise estatística foi utilizado o teste de Quiquadrado. Quando os pressupostos não foram satisfeitos foi aplicado o teste exato de Fisher.

\section{RESULTADOS}

Das 1734 colecistectomias laparoscópicas, 99 necessitaram de conversão para laparotomia, o que representa uma taxa de $5,7 \%$.

O principal motivo que levou à conversão foi a presença de inflamação e aderência entre as estruturas do pedículo

1. Residente de Cirurgia Geral do Hospital Governador Israel Pinheiro.

2. Cirurgião Geral; Coordenador do programa de Residência em Cirurgia Geral do Hospital Governador Israel Pinheiro.

3. Cirurgião Geral Hospital Governador Israel Pinheiro.

4. Cirurgião Geral Chefe do Serviço de Cirurgia Geral do Hospital Governador Israel Pinheiro.

Recebido em 07/02/2007

Aceito para publicação em 09/04/2007

Conflito de interesses: nenhum

Fonte de financiamento: nenhuma

Trabalho realizado no Hospital Governador Israel Pinheiro - IPSEMG- Belo Horizonte - MG. 
biliar com 42,53\%. Outras causas foram: coledocolitíase com $17,24 \%$, lesão da árvore biliar com 9,19\%, sangramento incontrolável com 4,59\%, vesícula escleroatrófica com 5,74\%. Falha de equipamento, anomalias anatômicas, dificuldade de acesso e fístula colecistoentérica, juntos representaram 20,69\% das conversões (Tabela 1).

A taxa de conversão das cirurgias eletivas foi de $5,44 \%$, enquanto a taxa das cirurgias de urgência foi de $14 \%$. $(\mathrm{p}=0,02)$ (Tabela 2).

Entre os pacientes com necessidade de conversão $71,43 \%$ tiveram procedimento com duração maior que 120 minutos, sendo que nos pacientes com procedimento integralmente laparoscópico apenas em 8,2\% a operação se estendeu além deste tempo $(\mathrm{p}<0,01)$.

Dos 1292 pacientes com menos de 60 anos de idade, em $57(4,41 \%)$ houve necessidade de conversão. Nos pacientes maiores de 60 anos em $42(11,11 \%)$ dos 378 operados, foi necessária a conversão para cirurgia aberta. $(\mathrm{p}=0,01)$ (Tabela 3$)$.

Foram operados 1466 pacientes do sexo feminino e 268 pacientes do sexo masculino. A taxa de conversão entre os pacientes do sexo masculino foi de $7,46 \%$, e a do sexo

Tabela 1 - Motivos de Conversão em 1734 colecistectomias vídeo-laparoscópica no Hospital Governador Israel Pinheiro.

\begin{tabular}{lrc}
\hline Motivo de conversão & N & N Percentual \\
\hline Inflamação/aderência & 37 & 42,53 \\
Coledocolitíase & 15 & 17,24 \\
Suspeita ou lesão biliar & 8 & 9,19 \\
Sangramento & 4 & 4,59 \\
V. escleroatrófica & 5 & 5,74 \\
Outros & 18 & 20,69 \\
TOTAL & 99 & 100 \\
\hline
\end{tabular}

Tabela 2 - Incidência de conversão das Colecistectomias Vídeo-laparoscópicas quanto ao caráter eletivo ou de urgência em 1734 pacientes.

\begin{tabular}{lrrr}
\hline & \multicolumn{1}{c}{ Eletiva } & Urgência \\
\hline Não-convertidas & 1671 & 50 & \\
Convertidas & $91 \quad(5,44 \%)$ & 7 & $(14 \%)$ \\
Total & 1762 & 57 & \\
\hline
\end{tabular}

$p=0,02$ feminino foi de 5,39\%, não havendo significância estatística $(\mathrm{p}=0,178)$ (Tabela 4).

Analisando-se os dados sobre o risco cirúrgico dos pacientes operados, 720 foram classificados como ASA I, 969 como ASA II e 45 como ASA III, sendo a taxa de conversão nos pacientes ASA I de 4,58\%, e nos pacientes ASA II e ASA III de $6,4 \%$ e $13,3 \%$, respectivamente.

A taxa de conversão global dos pacientes analisados foi de $5,7 \%$, enquanto nos pacientes com coledocolitíase foi de $25 \%$. (Tabela 5)

Apenas 10,8\% dos pacientes com operação integralmente laparoscópica permaneceram internados por tempo maior que 48 horas. Nos pacientes convertidos esse percentual foi de $79,31 \%$. $(p<0,01)$

A taxa de conversão do serviço permaneceu estável nos anos estudados variando de $6,3 \%$ no ano de 2000 a 5,24\% no ano de 2004.

Entre os pacientes com necessidade de conversão $7,14 \%$ evoluíram com íleo prolongado versus $0,93 \%$ no grupo de comparação (Fisher p=0,011). Quanto à infecção da ferida operatória $3,57 \%$ dos pacientes convertidos contra apenas $0,23 \%$ dos pacientes com procedimento integralmente laparoscópico (Fisher $\mathrm{p}=0,021$ ) evoluíram com essa complica-

Tabela 3 - Taxa de Conversão dos pacientes submetidos a colecistectomia vídeo-laparoscópica no Hospital Governador Israel Pinheiro, divididos em maiores e menores de 60 anos.

\begin{tabular}{|c|c|c|c|}
\hline & Convertidos & Não-convertidos & Total \\
\hline Menos de 60 anos & $57 \quad(4,41 \%)$ & 1235 & 1292 \\
\hline Mais de 60 anos & $42(11,11 \%)$ & 336 & 378 \\
\hline Total & 99 & 1571 & 1670 \\
\hline
\end{tabular}

$p=0,01$

Tabela 4 - Taxa de Conversão dos pacientes submetidos a Colecistectomia vídeo-laparoscópica no Hospital Governador Israel Pinheiro em relação ao sexo.

\begin{tabular}{lrrr}
\hline & Masculino & \multicolumn{2}{c}{ Feminino } \\
\hline Não-convertidos & 248 & 1387 & \\
Convertidos & $20 \quad(7,46 \%)$ & $79 \quad(5,39 \%)$ \\
Total & 268 & 1466 & \\
\hline
\end{tabular}

$p=0,178$

Tabela 5 - Tempo de permanência hospitalar dos pacientes submetidos a colecistectomia vídeo-laparoscópica no Hospital Governador Israel Pinheiro.

\begin{tabular}{lcrr}
\hline & $\begin{array}{c}\text { Alta em menos } \\
\text { de 48 horas }\end{array}$ & $\begin{array}{c}\text { Alta em mais } \\
\text { de 48 horas }\end{array}$ & Total \\
\hline Convertidos & $18(20,69 \%)$ & $69(79,31 \%)$ & $87(100 \%)$ \\
Não-convertidos & $1324(89,2 \%)$ & $161(10,8 \%)$ & $1485(100 \%)$ \\
\hline
\end{tabular}


ção. Entre os pacientes convertidos, 9,8\% apresentaram fístula biliar enquanto entre os pacientes não convertidos apenas $0,55 \%$. (Fisher $\mathrm{p}=0,001$ )

Ocorreram dois óbitos neste estudo, ambos devido a complicações cardiovasculares (Infarto Agudo do Miocárdio), um entre os pacientes convertidos e o outro nos pacientes com cirurgia laparoscópica. A taxa de mortalidade entre os pacientes convertidos se aproxima de $1 \%$, entretanto a mortalidade geral foi de $0,001 \%$.

\section{DISCUSSÃO}

As vantagens da colecistectomia laparoscópica amplamente comprovadas tornaram-na o procedimento de escolha para tratamento da colelitíase sintomática ${ }^{2}$. Algumas colecistectomias laparoscópicas, entretanto, necessitam de conversão. Na maioria dos casos, essa não é uma falha, nem uma complicação da operação, trata-se, na verdade, de medida necessária a fim de se evitar ambas ${ }^{3}$.

A taxa de conversão neste estudo foi de 5,7\%. Kama et $a l^{4}$ relatam que a taxa de conversão em diferentes hospitais varia entre $2 \%$ a $15 \%$, com média aproximada de $5 \%$, taxa esta atingida nos últimos anos com a maior experiência com a técnica operatória 5 .

A principal causa de conversão é a inflamação e a aderência entre as estruturas do triângulo de Calot ${ }^{6,20}$. Em nosso estudo $42,5 \%$ das conversões foram devidas a este fato. A inflamação e a aderência frustram a visualização da anatomia ductal biliar à laparoscopia, motivo que leva a um maior índice de conversão nestes casos $^{7}$.

O tempo operatório foi significativamente maior nos pacientes com necessidade de conversão. Rosen et $a l^{2}$ defendem que uma política de conversão precoce diante de uma dissecção difícil diminuiria o tempo e a morbidade do procedimento. No estudo de Majeski $\mathrm{J}^{21}$, foi visto que a cada intervalo de 30 minutos adicionais ao procedimento operatório, ocorreu aumento nas complicações intra-operatórias e pós-operatórias locais e sistêmicas.

A colecistite aguda doença que acarreta alterações da anatomia do sistema biliar devido ao intenso processo inflamatório, é aceita como fator preditor de conversão ${ }^{8,9}$. Nesse estudo esta condição esteve associada a uma taxa de conversão de $13,5 \%$ X 5,7\% do total $(p<0,01)$. O risco de conversão no paciente com colecistite aguda é dependente do momento da operação em relação ao início dos sintomas, pois é aceito por alguns autores ${ }^{10-12,25}$ que a operação nas primeiras 72 horas possui menor risco de conversão e de complicações. No estudo de Madan et $a l^{13}$ não houve necessidade de conversão nas operações realizadas nas primeiras 72 horas, enquanto que naquelas realizadas após 72 horas, a taxa de conversão foi igual a $29 \%$. Resultado similar ao encontrado por Eldar et $a l^{14}$. Entretanto no estudo de Knight et $a l,{ }^{15}$ não houve diferença na taxa de conversão nos pacientes operados nas primeiras 72 horas ou após este período. Resultados de estudos comparativos não randomizados entre operação precoce e tardia na mesma internação são conflitantes, mas tendem a indicar um aumento nas conversões no grupo de operação tardia, mas sem aumento na morbidade ${ }^{10,13,14,16}$. Em nosso estudo não foi possível a realização dessa análise.
No estudo de Bingener-Casey et $a l^{17}$ numa análise retrospectiva de 10 anos, observou-se forte tendência de queda na taxa de conversão dos pacientes com colecistite aguda, inicialmente $26 \%$ deles necessitavam conversão, ao término do estudo apenas $1 \%$ desses pacientes necessitou de conversão.

A lesão da via biliar é uma complicação grave e bastante temida pelos cirurgiões que transforma um procedimento cirúrgico de baixa morbi-mortalidade em um procedimento de alta morbi-mortalidade ${ }^{18}$. Após um aumento no número de lesões da via biliar com o advento da laparoscopia de $0,1 \%$ para $0,5 \%$, nota-se uma tendência à redução desse índice, devido principalmente à maior experiência com a técnica ${ }^{6,19}$.

Em nosso estudo houve oito casos de lesão da via biliar o que representa $0,46 \%$. As lesões de via biliar foram diagnosticadas no per-operatório com ou sem o auxílio da colangiografia intra-operatória. Em todos esses pacientes foi necessária a laparotomia e a correção da lesão no mesmo ato foi a terapêutica preferida.

A principal causa de lesão é a má-identificação da anatomia biliar (70-80\% das lesões). Hunter et $a l^{20}$ defendem que o uso de laparoscópio de $30^{\circ}$, dissecção próxima à junção infundíbulo-cística e a conversão para cirurgia aberta quando houver incerteza, pode prevenir a lesão da via biliar.

Em estudo brasileiro multicêntrico conduzido por Savassi-Rocha ${ }^{18}$ com mais de 90.000 pacientes foi encontrada incidência de $0,18 \%$ de lesões da árvore biliar. Em Serviços com volume menor que 50 cirurgias a incidência de lesão foi de $0,77 \%$, em contraste a incidência nos serviços com mais de 500 procedimentos foi de $0,16 \%$.

É aceito que a idade acima dos 60 anos é um fator de risco para conversão ${ }^{21}$.

Nossos dados mostram que entre os pacientes com menos de 60 anos 4,4\% necessitaram de conversão, enquanto entre os pacientes com mais de 60 anos houve $11,1 \%$ de conversão. $(\mathrm{p}=0,01)$. O risco aumentado de conversão, no paciente idoso, se deve provavelmente ao fato da idade sugerir uma história longa de cálculos na via biliar e um número maior de colecistites prévias ${ }^{4,22}$.

Vários estudos identificaram o sexo masculino como fator de risco para conversão, provavelmente pela associação mais freqüente com doenças mais graves tanto aguda quanto crônica ${ }^{16}$.

Livingston et al ${ }^{5}$ demonstraram que homens obesos com colecistite tanto aguda quanto crônica tiveram duas vezes maior risco de conversão (12,9\%) que mulheres (5,3\%). Em nosso estudo este fato não foi confirmado sendo a taxa de conversão entre os pacientes do sexo masculino de 7,46\%, e a do sexo feminino de 5,39\%, não havendo significância estatística $(\mathrm{p}=0,178)$.

O risco cirúrgico proposto pela American Society of Anesthesiology (ASA), também foi uma variável dessa análise retrospectiva. Dos 1734 pacientes submetidos à colecistectomia laparoscópica, a taxa de conversão em pacientes classificados como ASA I foi de 4,58\%, enquanto que aqueles dos grupos ASA II e ASA III foram 6,4\% e 13,3\%, respectivamente. Pacientes com co-morbidade sistêmica descompensada tiveram seu risco relativo para conversão 
cerca de duas vezes maior do que pacientes previamente hígidos ( $\mathrm{p}=0,026)$.

A necessidade de conversão aumenta o tempo de permanência hospitalar.

Este fato pode ser atribuído a um maior índice de complicações pós-operatórias, à dor mais intensa referida pelo paciente ${ }^{18}$ ou à própria característica da doença que levou à conversão, pois é sabido que determinadas doenças associadas a um maior índice de conversão, como por exemplo a colecistite aguda, necessitam uma permanência hospitalar mais prolongada, para continuação do tratamento do paciente. Dentre as operações exclusivamente laparoscópicas, 11,8\% dos pacientes tiveram alta hospitalar em menos de 48 horas. Em contrapartida, $79,31 \%$ dos pacientes convertidos tiveram alta hospitalar em mais de 48 horas ( $p<0,001)$.

Em nosso Serviço a taxa de conversão permaneceu estável nos anos estudados $(5,4 \%$ - 6,3\%) refletindo a experiência dos membros do Serviço com a colecistectomia vídeolaparoscópica. E apesar do fato de se tratar de um Serviço de Residência Médica as taxas de conversão permanecem baixas, devido à participação efetiva dos cirurgiões experientes nos procedimentos realizados. Fato similar ao encontrado por Bingener-Casey ${ }^{17} \mathrm{em}$ seu estudo.

Pacientes que necessitam conversão tendem a ter maior tempo operatório, alta morbidade e maior permanência hospitalar quando comparados àqueles com procedimento integralmente laparoscópico ${ }^{3}$. Em nosso estudo as complica- ções foram maiores nos pacientes convertidos no que diz respeito à infecção de ferida operatória, íleo prolongado e ocorrência de fístula biliar.

Os principais motivos que levaram à conversão foram: a presença de inflamação e aderência entre as estruturas do pedículo biliar, coledocolitíase, suspeita ou lesão da árvore biliar, sangramento incontrolável e vesícula escleroatrófica.

Os fatores associados a uma maior chance de conversão, identificados por esse estudo são: operação em caráter de urgência, idade avançada (maior de 60 anos), pacientes com colecistite aguda e vesícula escleroatrófica.

A duração do ato operatório foi significativamente maior nos pacientes com necessidade de conversão para operação aberta. Esse fato pode significar um atraso na decisão de conversão do procedimento.

A colecistectomia laparoscópica mostrou-se um procedimento seguro no grupo analisado, com baixo índice de conversão $5,7 \%$. O motivo mais freqüente para interrupção do procedimento laparoscópico foi a ocorrência de inflamação no pedículo biliar e a aderência entre as estruturas.

A identificação dos fatores associados a um maior índice de conversão possibilita à equipe cirúrgica estimar o grau de dificuldade do procedimento, preparando melhor o paciente para o risco de conversão e permitindo a participação de um cirurgião mais experiente num procedimento de maior risco.

\begin{abstract}
Background: This study has the objective to evaluate factors implied with conversion from laparoscopic to open procedures. Method: We retrospectively analyzed the patients' records submitted to laparoscopic cholecystectomy, from January 2000 to December 2004. Results: One thousand seven hundred and thirty four laparoscopic cholecystectomies were performed during this period. The total conversion rate was $5.7 \%$, scheduled surgery was $5.44 \%$ and emergency surgery was $14 \%(p=0.019)$. Male patients had a conversion rate of $7.46 \%$ and female patients had a conversion rate of $5.39 \%$. $(p=0.178)$. Patients older than 60 need conversion in $11.11 \%$, and among patients younger than 60 , the rate was $4.41 \%$. $(p=0.01)$. The main cause of conversion was inflammation/adherence in the structures of the gallbladder pedicle, and was responsible for $42.53 \%$, choledocholithiasis accounted for $17.24 \%$, and suspicion or bile duct injury corresponded to 9.19\%. An operative time longer than 120 minutes was observed in $71.43 \%$ of converted patients, and $8.2 \%$ of nonconverted ones. In the converted group, $79.31 \%$ were discharged more than 48 hours after surgery, and in the nonconverted group, $10.8 \%$ were discharged after this period. $(p=0,001)$. Conclusion: The risk factor observed in this study was age older than 60 years, emergency surgery and acute cholecystitis. The identification of these factors can help estimate the difficulty of the procedure and permit the surgeon to give better information to the patients about the risk of conversion.
\end{abstract}

Keywords: Cholecystectomy; Laparoscopic; Riskfactors.

\section{REFERÊNCIAS}

1. Savassi-Rocha PR. Colecistectomia videolaparoscópica. In: Pereira-Lima L. Condutas em cirurgia hepatobiliopancreática. $1^{a}$ ed. Rio de Janeiro: Medsi; 1995. p.139-71.

2. Rosen M, Brody F, Ponsky J. Predictive factors for conversion of laparoscopic cholecystectomy. Am J Surg. 2002;184(3):2548.

3. Alponat A, Kum CK, Koh BC, Rajnakova A, Goh PM. Predictive factors for conversion of laparoscopic cholecystectomy. World J Surg. 1997;21(6):629-33.
4. Kama NA, Kologlu M, Doganay M, Reis E, Atli M, Dolapci M. A risk score for conversion from laparoscopic to open cholecystectomy. Am J Surg. 2001;181(6):520-5.

5. Livingston $E H$, Rege RV. A nationwide study of conversion from laparoscopic to open cholecystectomy. Am J Surg. 2004;188(3):205-11.

6. Connor S, Garden OJ. Bile duct injury in the era of laparoscopic cholecystectomy. Br J Surg. 2006;93(2):158-68.

7. Bingener J, Richards ML, Schwesinger WH, Strodel WE, Sirinek KR. Laparoscopic cholecystectomy for elderly patients: gold standard for golden years? Arch Surg. 2003;138(5):531-5; discussion 535-6. 
8. Simopoulos C, Botaitis S, Polychronidis A, Tripsianis G, Karayiannakis AJ. Risk factors for conversion of laparoscopic cholecystectomy to open cholecystectomy. Surg Endosc. 2005;19(7):905-9. Epub 2005 May 4.

9. Bulbuller N, Ilham YS, Baktir A, Kirkill C, Dogru O. Implementation of a scoring system for assessing difficult cholecystectomy in a single center. Surg Today. 2006;36(1):3740.

10. Bhattacharya D, Senapati PS, Hurle R, Ammori BJ. Urgent versus interval laparoscopic cholecystectomy for acute cholecystitis: a comparative study. J Hepatobiliary Pancreat Surg. 2002;9(5):53842.

11. Brodsky A, Matter I, Sabo E, Cohen A, Abrahamson J, Eldar S. Laparoscopic cholecystectomy for acute cholecystitis: can the need for conversion and the probability of complications be predicted? A prospective study. Surg Endosc. 2000;14(8):75560.

12. Tayeb M, Raza SA, Khan MR, Azami R. Conversion from laparoscopic to open cholecystectomy: multivariate analysis of preoperative risk factors. J Postgrad Med. 2005;51(1):17-20; discussion 21-2.

13. Madan AK, Aliabadi-Wahle S, Tesi D, Flint LM, Steinberg SM. How early is early laparoscopic treatment of acute cholecystitis? Am J Surg. 2002;183(3):232-6.

14. Eldar S, Sabo E, Nash E, Abrahamson J, Matter I. Laparoscopic cholecystectomy for acute cholecystitis: prospective trial. World J Surg. 1997;21(5):540-5.

15. Knight JS, Mercer SJ, Somers SS, Walters AM, Sadek SA, Toh SK. Timing of urgent laparoscopic cholecystectomy does not influence conversion rate. Br J Surg. 2004;91(5):601-4.

16. Tang B, Cuschieri A. Conversions during laparoscopic cholecystectomy: risk factors and effects on patient outcome. J Gastrointest Surg. 2006;10(7):1081-91.
17. Bingener-Casey J, Richards ML, Strodel WE, Schwesinger WH, Sirinek KR. Reasons for conversion from laparoscopic to open cholecystectomy: a 10-year review. J Gastrointest Surg. 2002;6(6):800-5.

18. Savassi-Rocha PR, Almeida SR, Sanches MD, Andrade MA, Frerreira JT, Diniz MT, Rocha AL. Iatrogenic bile duct injuries. Surg Endosc. 2003;17(9):1356-61. Epub 2003 Jun 19.

19. Nuzzo G, Giuliante F, Giovannini I, Ardito F, D’Acapito F, Vellone M, Murazio M, Capelli G. Bile duct injury during laparoscopic cholecystectomy: results of an Italian national survey on 56.591 cholecystectomies. Arch Surg. 2005;140(10):986-92.

20. Hunter JG. Avoidance of bile duct injury during laparoscopic cholecystectomy. Am J Surg. 1991;162(1):71-6.

21. Majeski J. Laparoscopic cholecystectomy in geriatric patients. Am J Surg. 2004;187(6):747-50.

22. Lai PBS, Kwong KH, Leung KL, Kwok SP, Chan AC, Chung SC, Lau WY. Randomized trial of early versus delayed laparoscopic cholecystectomy for acute cholecystitis. B J Surg.1998;85(6):7647.

Como citar este artigo:

Lima EC, Queiroz FL, Ladeira FN, Ferreira BM, Bueno JGP, Magalhães EA. Análise dos fatores implicados na conversão da colecistectomia laparoscópica. Rev Col Bras Cir. 2007;34(5). Disponível em URL: www.scielo.br/rcbc

Endereço para correspondência:

Rua Professor Arduíno Bolívar, 453. Apartamento 01.

Bairro: Santo Antônio.

30350-140 - Belo Horizonte - MG.

E-mail: eniocorrealima@hotmail.com 